\title{
Teste de força de preensão manual: estudo da fadiga mioelétrica do flexor radial do carpo e flexor superficial dos dedos
}

CDD. 20.ed. 616.7

796.073

\author{
Marco Tulio BAPTISTA ${ }^{* * * *}$ \\ Fábio Alves MACHADO ${ }^{* *}$ \\ Glauber Ribeiro PEREIRA* \\ Jurandir NADAL* \\ Liliam Fernandes OLIVEIRA***
}

*Instituto Alberto Luiz Coimbra de PósGraduação e Pesquisa de Engenharia, Universidade Federal do Rio de Janeiro. ${ }^{* *}$ Instituto de Pesquisa da Capacitação Física do Exército.

***Escola de Educação

Física e Desportos, Universidade Federal do Rio de Janeiro.

Um protocolo de força de preensão manual (FPM) em degraus de intensidade foi empregado para estudo da fadiga dos músculos flexor radial do carpo (FRC) e flexor superficial dos dedos (FSD) por meio do registro da força de preensão sustentada. Foi feita a análise do sinal eletromiográfico de superfície destes músculos no domínio do tempo e da frequência de $2 \mathrm{kHz}$. Foi utilizado um dinamômetro eletrônico e um conversor analógico-digital de 16 bits. Participaram deste estudo 12 indivíduos saudáveis, ativos e destros, com média de idade $21,53 \pm 1,26$ anos, percentual de gordura $7,76 \pm 3,53 \%$, peso corporal $74,9 \pm 10,36 \mathrm{~kg}$ e estatura $180,69 \pm 7,14 \mathrm{~cm}$. Os indivíduos realizaram o teste de contração isométrica voluntária máxima (CIVM) da mão dominante seguido do protocolo de degraus submáximos em $20 \%$, $40 \%$ e $60 \%$ da CIVM por 10 segundos cada. 0 processamento dos sinais envolveu a filtragem passa banda e o cálculo dos valores de raiz média quadrática (RMS) e frequência mediana (FM) em cada degrau de contração submáxima. A análise de variância "two-way" foi aplicada para os valores de RMS e FM. 0 teste proposto não gerou queda do rendimento de força nos degraus submáximos estabelecidos e a instauração do processo de fadiga do FSD. Por outro lado, o FRC apresentou sinais de fadiga mioelétrica sugerindo o processo de falência da FPM. Estes dados sugerem que a fadiga mioelétrica dos flexores dos dedos durante a FPM é um processo tardio à fadiga dos estabilizadores do punho. 0 protocolo em degrau de $60 \%$ parece desencadear o processo de fadiga mioelétrica do músculo FRC, mas não do FSD, baseado na análise da ativação muscular nos domínios do tempo e frequência.

PalaVRAS-Chave: Isometria; Dinamometria; Mão; Eletromiografia.

\section{Introdução}

A mão é um órgão complexo sistema destinado a realizar uma multiplicidade de objetivos, combinando força e destreza ${ }^{1}$. Como órgão preênsil é capaz tanto de produzir altos níveis de força para sustentar cargas elevadas, como forças mínimas para manipular objetos em tarefas de precisão, necessárias em muitas atividades da vida diária ${ }^{2}$.

A força de preensão manual (FPM) é uma medida usada como preditora da força dos membros superiores $^{3-4}$ e aceita como um índice objetivo que representa a funcionalidade destes membros ${ }^{5}$. SMITH et al. ${ }^{6}$ encontraram relação direta entre a FPM e a força total do corpo em indivíduos idosos. Neste estudo, a força total do corpo seria o somatório de forças que podem ser produzidas por todos os segmentos corporais. FRY et al. ${ }^{4}$ também encontraram uma correlação entre a FPM e o desempenho em atletas juniores de levantamento de peso. Esta medida pode fornecer informações ao treinador sobre o estado de prontidão física do atleta $^{4}$, como também aos médicos e fisioterapeutas ${ }^{7}$ sobre a determinação de estratégias de tratamento ou os efeitos de diferentes procedimentos clínicos ${ }^{8}$. DEVRIES ${ }^{a}$ citado por ALKURDI e DwEIRI ${ }^{9}$ afirma que a média da FPM está correlacionada com a força total de 22 músculos do corpo, indicando deste modo a importância dessa medida. 
A eficiência do sistema nervoso central no controle da força de preensão pode ser estudada pelas mudanças nos níveis de força na realização de tarefas complexas ${ }^{10}$ envolvendo vários músculos. Durante a FPM, os músculos flexores da mão e do antebraço são os agonistas, enquanto os extensores de punho auxiliam na estabilização do o punho ${ }^{11}$. O flexor superficial dos dedos (FSD) é o agonista principal no movimento de preensão ${ }^{12}$, enquanto o flexor radial do carpo (FRC) é responsável pela flexão do punho, sendo ainda utilizado na sustentação da pegada nos movimentos explosivos e na preensão em agarras abauladas ${ }^{13}$.

Quando é necessária a manutenção da força de preensão máxima e/ou submáxima por um período de tempo, pode haver a instalação do processo de fadiga muscular. A fadiga tem sido frequentemente descrita como um processo fisiológico tempo dependente que engloba numerosos fatores centrais e periféricos ${ }^{14}$, afetando a capacidade de produção de força e prejudicando o desempenho ${ }^{15-16}$.

Um fato importante que está associado à produção de força é a fadiga neuromuscular. Isso em certas condições pode refletir numa diminuição do desempenho e/ou o ponto de falha em que o músculo não é mais capaz de sustentar a força requerida ou o nível de produção de trabalho ${ }^{17-21}$. Este fenômeno é conhecido como manifestação mioelétrica da fadiga ${ }^{22}$ e se traduz pela análise do sinal eletromiográfico por meio da interpretação dos valores da raiz quadrática média $(\mathrm{RMS})^{23}$, do sinal eletromiográfico integrado (IEMG) ${ }^{24}$ e do

\section{Método}

\section{Amostra}

Participaram deste estudo 12 indivíduos saudáveis, ativos e destros, com média de idade $21,53 \pm 1,26$ anos, percentual de gordura 7,76 \pm $3,53 \%$, peso corporal $74,9 \pm 10,36 \mathrm{~kg}$ e estatura $180,69 \pm 7,14 \mathrm{~cm}$ sem antecedentes de lesão do aparelho locomotor. Todos os indivíduos participam de treinamento físico regular e supervisionado visando ao condicionamento neuromuscular e cardiopulmonar. Foram excluídos da amostra sujeitos que apresentaram alguma desordem neuromuscular e/ou articular, no membro superior dominante, num período inferior a 18 meses. comportamento da potência das frequências média e mediana ${ }^{25}$ do sinal.

Terapeutas, médicos, engenheiros e técnicos esportivos estão interessados na medição da força de preensão manual a fim de estabelecer uma referência a partir do qual eles avaliem seu tratamento, possam projetar uma mão, bem como realizem uma avaliação do desempenho de atletas? Porém, para estabelecer estas referências é necessário analisar em níveis de força submáximos e associados ao comportamento eletromiográfico (EMG) dos músculos envolvidos. Contudo, testes de força de preensão manual em degraus submáximos aplicados ao estudo da fadiga mioelétrica dos músculos flexor radial do carpo e flexor superficial dos dedos são raros de serem encontrados na literatura.

LARIVIERE et al. ${ }^{26}$ propuseram um protocolo baseado em medidas de EMG, com o intuito de avaliar o efeito do uso de dois tipos de luvas sobre a sobrecarga dos músculos do antebraço. Os autores sugerem que níveis de esforço considerados muito altos (superiores a 60\% da CIVM) podem apresentar diferença na fadiga muscular de músculos sinergistas, devido a uma diferença na oclusão do fluxo sanguíneo intra-muscular.

Desta forma, o objetivo deste trabalho foi aplicar um protocolo em degraus submáximos de preensão manual para análise da instalação do processo de fadiga dos músculos flexor radial do carpo (FRC) e flexor superficial dos dedos (FSD), baseado na hipótese de que a força de preensão manual próxima a $60 \%$ poderá provocar a fadiga nos músculos analisados.
O protocolo experimental seguiu as Normas de Realização de Pesquisa em Seres Humanos, Resolução número 196/96 do Conselho Nacional de Saúde, todos os voluntários assinaram o termo de consentimento livre e esclarecido e aprovado pelo comitê de ética local.

\section{Avaliações}

O cálculo do percentual de gordura foi realizado por um avaliador experiente através da plicometria, fazendo-se uso do protocolo de JACKSON \& POLLOCK $^{27}$ de três dobras cutâneas (peitoral, abdominal e coxa) e com três medidas em cada uma destas. $\mathrm{O}$ adipômetro utilizado foi o da marca Lange ${ }^{\circledR}$ Skinfold Caliper 
(com pressão constante de $10 \mathrm{~g} / \mathrm{mm}$ e precisão de $0,5 \mathrm{~mm}$ com variação de $0,1 \mathrm{~mm}$ ).

Foi utilizado um dinamômetro eletrônico de força de preensão manual, modelo TRF200 (EMG System do Brasil, São José dos Campos, SP, Brasil) com cabo de $2 \mathrm{~m}$ flexível, blindado e um sistema de aquisição de dados que permitisse a leitura deste equipamento até 200 kgf. Para a aquisição do sinal EMG, utilizou-se eletrodos de $\mathrm{Ag} / \mathrm{AgCl}$ em uma configuração bipolar segundo o protocolo proposto pelo SENIAN ${ }^{28}$.

O sistema de aquisição foi composto de um computador (Pentium - $200 \mathrm{MHz}$ ) e um conversor analógico-digital (EMG System do Brasil, São José dos Campos, SP, Brasil) de 16 bits e faixa dinâmica de $\pm 5 \mathrm{~V}$. A frequência de amostragem foi de $2 \mathrm{kHz}$. Os eletrodos de $\mathrm{Ag}-\mathrm{AgCl}$ foram posicionados sobre os músculos Flexor Superficial dos Dedos e do Flexor Radial do Carpo do braço direito, conforme as recomendaçōes do SENIAM e também por anatomia palpatória ${ }^{29}$, após a preparação da pele ${ }^{28}$. O eletrodo de referência foi posicionado sobre o olécrano do cotovelo esquerdo.

\section{Protocolo}

Os indivíduos foram posicionados em pé, com o membro superior em extensão, ao longo do corpo, com leve abdução de ombro e punho em posição neutra. Esta posição é vantajosa por apresentar os mais altos valores de preensão manual ${ }^{30}$. $\mathrm{O}$ procedimento experimental foi dividido em três etapas descritas a seguir:

Etapa 1 - Experimentação pré-teste: $\mathrm{O}$ teste foi precedido por um processo de experimentação, que consistiu de contrações submáximas de força de preensão manual (FPM) seguidas por uma tentativa de esforço máximo;

Etapa 2 - Normalização: Com a finalidade de determinar os valores da contração isométrica voluntária máxima (CIVM); e

Etapa 3 - Teste de degraus submáximos com "feedback" visual: Nesta etapa fez-se uso de um monitor de computador colocado a $75 \mathrm{~cm}$ do indivíduo. Os degraus de contração submáxima foram em 20\% (R2_1 e R2_2), 40\% (R4_1 e R4_2) e 60\% (R6_1 e R6_2) da CIVM. Cada degrau teve duração de $10 \mathrm{~s}$, com intervalo de repouso de 15 s entre os degraus de mesma intensidade e $45 \mathrm{~s}$ entre as diferentes intensidades. $\mathrm{O}$ teste total teve a duração de 205 s. Cada indivíduo realizou duas vezes a CIVM de preensão da mão dominante com duração de 6 s e 2 min de intervalo entre cada tentativa. O maior valor das duas CIVM em kgf foi selecionado para análise.

\section{Aquisição e processamento dos sinais}

Os sinais de EMG foram pré-processados utilizando um filtro Butterworth passa-banda de $4^{\mathrm{a}}$ ordem $(20-400 \mathrm{~Hz})$ e para a redução das frequências de interferência $(60 \mathrm{~Hz})$ e seus harmônicos foi utilizado um filtro "notch" de $2^{\text {a }}$ ordem ${ }^{31}$. Ambos os filtros foram aplicados nos sentidos direto e reverso com o intuito de remover as distorçōes de fase. $\mathrm{O}$ sinal de EMG foi subamostrado para $1 \mathrm{kHz}$ devido ao custo computacional e normalizado pelo valor máximo da CVIM.

A partir do sinal filtrado, foram calculados os valores RMS a cada 500 amostras do trecho de $7 \mathrm{~s}$ de cada degrau (a partir do segundo até o penúltimo segundo), totalizando 14 valores por degrau. Os valores médios de RMS e FM foram calculados para cada degrau, sendo que o RMS foi normalizado (RMS_N). A FM foi calculada pelo método do espectograma pela Short-Time Fourier Transform (STFT). Os valores de força relativa também foram calculados para o mesmo intervalo, para determinar se o indivíduo permaneceu nos níveis desejados de intensidade durante o teste. As rotinas para processamento foram desenvolvidas com o aplicativo Matlab7.0 (The Mathworks, EUA).

\section{Análise estatística}

A análise estatística foi realizada no programa Statistica 7.0. A distribuição normal dos dados foi testada pelo teste Shapiro-Wilk. A análise de variância "twoway" (ANOVA para três de graus de intensidades, duas tentativas por intensidade) para cada músculo analisado e o teste "post hoc" Tukey para verificar diferenças significativas. A variabilidade dos valores de RMS e FM foi calculada pelo coeficiente de variação (CV). O nível de significância foi de $\mathrm{p}<0,05$. 


\section{Resultados}

A contração voluntária isométrica máxima (CVIM) média encontrada foi 29,09 \pm 8,40 kgf.

A FIGURA 1 mostra um exemplo do protocolo total aplicado. Nota-se nesta figura que há um nível mínimo de preensão manual necessário para a sustentação do equipamento em situação de repouso. Não foi observada diferença significativa nas forças relativas entre as tentativas de cada degrau de contração submáxima em 20\%, 40\% e 60\% da CIVM (FIGURA 2), sendo que os indivíduos foram capazes de manter o torque solicitado.

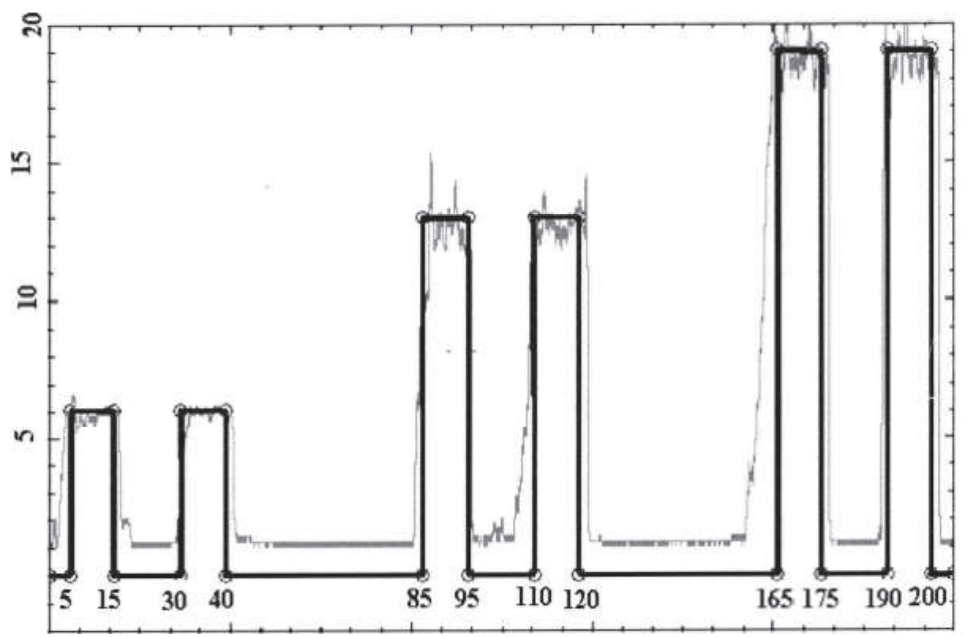

FIGURA 1 - Degraus de contração submáxima em 20\%, 40\% e 60\% da CIVM. Cada degrau teve duração de 10 s, com intervalo de $15 \mathrm{~s}$ entre os degraus de mesma intensidade e $45 \mathrm{~s}$ entre as diferentes intensidades.

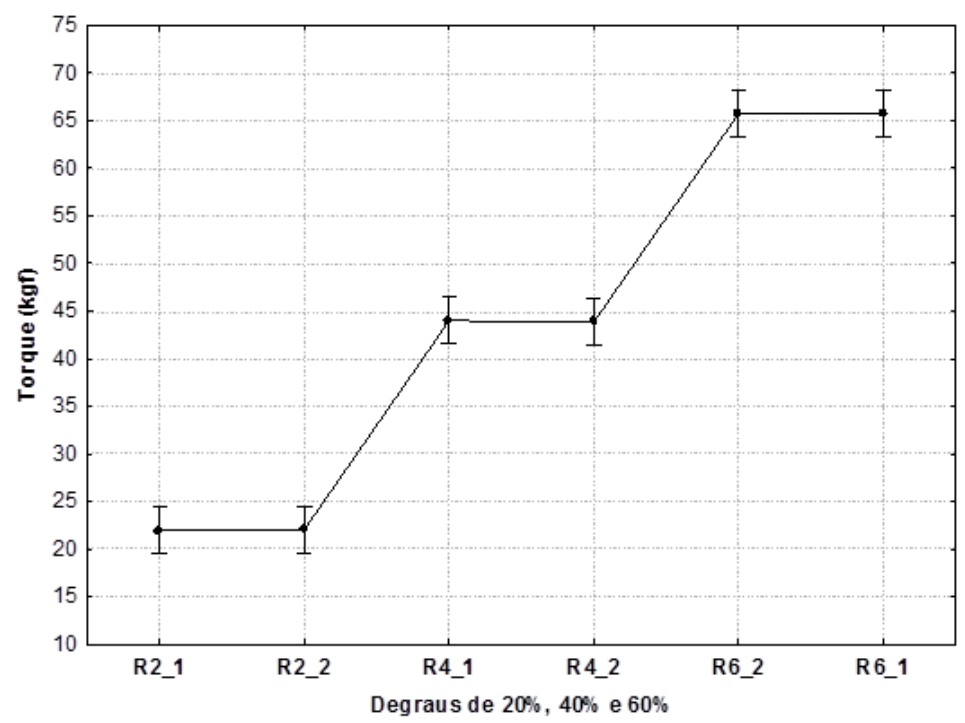

FIGURA 2 - Força relativa nos degraus de 20\% (R2_1 e R2_2), 40\% (R4_1 e R4_2) e 60\% (R6_1 e R6_2).

Para a FM do FSD não houve diferença significativa entre os degraus de mesma intensidade (FIGURA 3). Para o FRC, as duas tentativas do último degrau de 60\% apresentaram valores de FM significativamente menores $(p<0,05)$ do que os dos degraus anteriores.
Para ambos os músculos, houve aumento significativo $(\mathrm{p}<0,05)$ dos valores de RMS normalizados (RMS_N) entre os degraus de intensidade analisados (FIGURA 4). Porém, não houve diferença significativa entre as tentativas por degrau. 


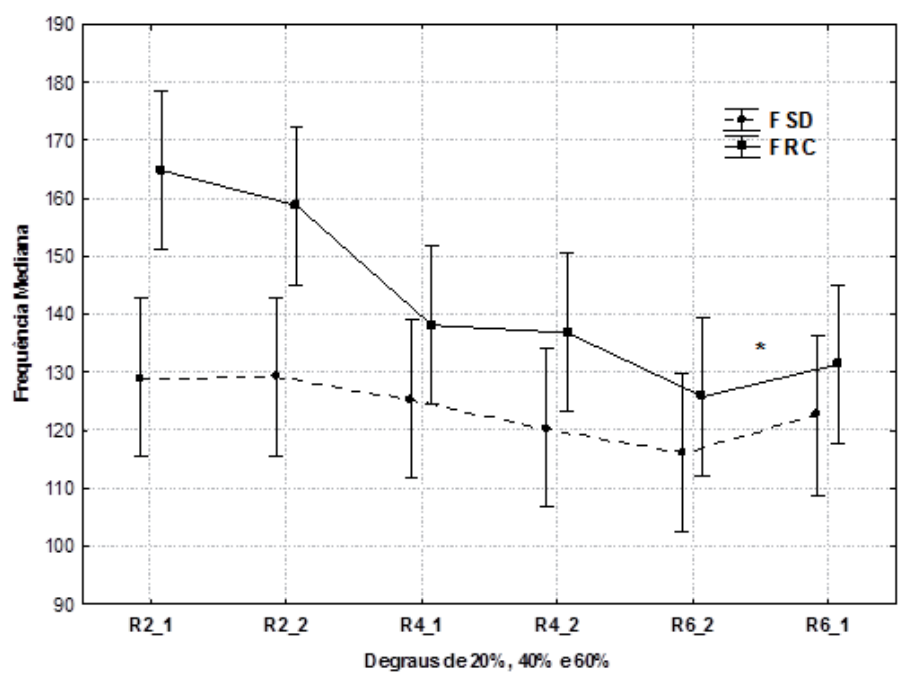

${ }^{*} p<0,05$, para o FRC, R6 1 e R6 2 apresentaram valores de FM significativamente menores do que os dos degraus de $20 \%$ e $40 \%$.

FIGURA 3 - FM do Flexor Superficial dos Dedos (FSD) e Flexor Radial do Carpo (FRC) nos degraus de 20\% (R2_1 e R2_2), 40\% (R4_1 e R4_2) e 60\% (R6_1 e R6_2).

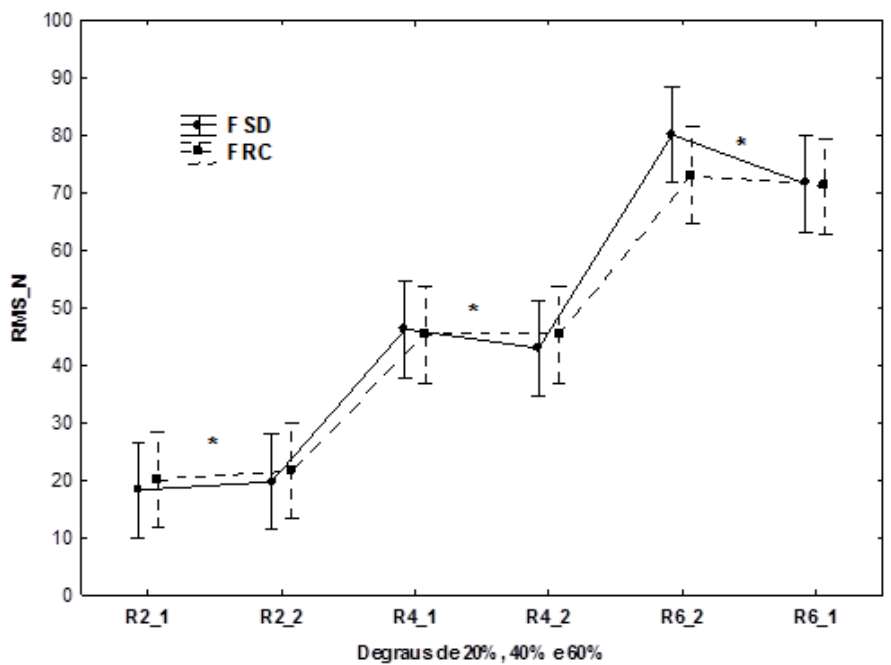

${ }^{*} p<0,05$, para ambos os músculos, houve aumento significativo dos valores de RMS normalizados (RMS_N) entre os degraus de $20 \%, 40 \%$ e $60 \%$.

FIGURA 4 - RMS_N do Flexor Superficial dos Dedos (FSD) e Flexor Radial do Carpo (FRC) nos degraus de 20\% (R2_1 e R2_2), 40\% (R4_1 e R4_2) e 60\% (R6_1 e R6_2).

\section{Discussão}

AlKurdi e DWEIRI ${ }^{9}$ utilizaram uma amostra de 20 estudantes universitários jordanianos e um transdutor de força eletrônico diferente e com empunhadura distinta. Embora existisse estas diferenças técnicas, os valores de CVIM encontrados no presente estudo $(29,09 \pm 8,40 \mathrm{kgf})$ foram próximos aos evidenciados por ALKURDI e DwEIRI ${ }^{9}$ $(30,67 \pm 8,67 \mathrm{kgf})$.

$\mathrm{O}$ presente estudo aponta que o teste de degraus submáximos de preensão manual não resultou em redução do torque sustentado nos diferentes degraus. Contudo, houve a manifestação miolétrica da fadiga do músculo FRC.
O aumento da atividade eletromiográfica em contraçôes isométricas ocorre à medida que a tensão muscular se eleva ${ }^{30}$, refletindo o recrutamento de unidades motoras suplementares, necessário para compensar a diminuição da força de contração que ocorre na fadiga das fibras musculares resultando em aumento da amplitude EMG ${ }^{25}$.

A análise espectral deve ser utilizada conjuntamente ao RMS para interpretar o comportamento da resposta das unidades motoras diante de tarefas repetitivas, particularmente nas que envolvem contraçôes isométricas $^{32}$. Os índices da frequência média e a frequência mediana, fornecidas pelo 
espectro de potência, estão relacionados com a velocidade de condução do estímulo por parte da fibra muscular e alterações na sincronização e na frequência de disparo das unidades motoras ${ }^{33}$. Portanto, a frequência mediana é indicada para analisar a fadiga muscular por ser menos sensível aos ruídos e mais sensível aos processos bioquímicos e fisiológicos que ocorrem dentro dos músculos durante as contraçóes sustentadas ${ }^{18}$.

Neste contexto, nossos resultados indicam que o músculo FSD parece não apresentar fadiga mioelétrica, uma vez que os valores de FM não reduziram concomitantemente com o aumento da RMS nos degraus estabelecidos. Este fato pode explicar a não alteração dos platôos de torque nos percentuais de CVIM avaliados (FIGURA 2). E também sugere que este músculo poderia ser solicitado em maiores níveis de esforço neuromuscular, tanto na duração do tempo de isometria, quanto no percentual de esforço.

Por outro lado, o FRC apresentou simultaneamente redução na FM correspondente aos degraus de $60 \%$ e aumento na amplitude ao longo dos degraus de $20 \%$ a $60 \%$. Este comportamento sugere que o processo de instauração de fadiga mioelétrica, sinalizado a partir de $60 \%$ da CIVM do FRC passa a comprometer o gesto motor de atividades esportivas ou laborais que envolvam a força de preensão manual por um tempo igual ou superior a $6 \mathrm{~s}$ de isometria. LARIVIERE et al. ${ }^{26}$ reforçam os nossos achados, ao sugerir que níveis de esforço considerados muito altos (60\% da CIVM) podem apresentar diferença na fadiga muscular de músculos sinergistas, devido a uma diferença na oclusão do fluxo sanguíneo intra-muscular.

HOOZEMANS e VAN DIEËN ${ }^{34}$ estudaram a contribuiçãao de seis músculos na preensão manual, sendo que dois deles são o FRC e o FSD. Observaram que o FRC foi um dos músculos que exerceu menor influência na determinação da força máxima de preensão palmar. Em termos cinesiológicos, os dois músculos analisados apresentam funções distintas. O FSD e o flexor profundo dos dedos são os músculos agonistas que imprimem maior força no movimento de preensão ${ }^{12}$. Para que a FPM seja máxima é necessária uma estabilização do punho, realizada pelos músculos extensores e flexores desta articulação. Neste estudo, foi mostrado que o FRC apresentou sinais de fadiga no último degrau de esforço representada pela redução da frequência mediana. Contudo, a fadiga mioelétrica do músculo FRC não acarretou queda do desempenho. O estudo da estabilização do punho pode ser mais especificamente investigado em futuros experimentos com a aquisição simultânea do EMG dos músculos flexores e extensores do carpo.

A ação sinérgica dos músculos flexores e extensores dos dedos e a interação de grupos musculares é um fator importante na força de preensão ${ }^{8}$. Dentro do conceito de "force sharing" ou "torque sharing" 35 , o padrão de ativação individual dos músculos dentro de um grupamento e entre sinergistas, pode variar. Este fato tem sido evidenciado em várias situaçōes ${ }^{35-36}$. Dessa forma, é possível que a função estabilizadora do FRC tenha resultado em processo de fadiga, enquanto o agonista FSD, não.

A análise dos padrões de ativação dos músculos estabilizadores do punho, no teste proposto, deverá incluir a análise do músculo extensor radial do carpo. Este fato foi uma limitação do presente estudo, uma vez que este músculo age sinergicamente na contração isométrica da extensão do punho a fim de evitar a flexão do punho, movimento este indesejável pelo fato de reduzir a força exercida pelos flexores dos dedos.

O teste proposto não gerou queda do rendimento de força nos degraus submáximos estabelecidos, bem como a instauração do processo de fadiga do FSD. Por outro lado, o FRC apresentou sinais de fadiga mioelétrica sugerindo o processo de falência da estabilização do punho, demonstrando que a fadiga mioelétrica dos flexores dos dedos durante a preensão manual pode ser um processo tardio à fadiga dos estabilizadores do punho.

Com base na ativação muscular nos domínios do tempo e frequência, o degrau $60 \%$ parece desencadear o processo de fadiga mioelétrica do FRC, mas não do FSD.

\section{Nota}

a. DeVries HA. Physiology of exercise in physical education and athletics. Dubuque: Brown; 1980. 


\begin{abstract}
Handgrip strength test: a study of myoelectric fatigue of flexor carpi radialis and the flexor digitorum superficialis

A test protocol of handgrip strength in steps was used in order to study the fatigue of the flexor carpi radialis (FRC) and the flexor digitorum superficialis (FSD) muscles with signal processing in the time and frequency domains. It was used an electronic hand grip dynamometer, and a 16-bits analog-digital converter. The sampling frequency was $2 \mathrm{kHz}$. The study included 12 healthy subjects, active and righthanded, mean age $21.53 \pm 1.26$ years, body fat percentage $7.76 \pm 3.53 \%$, weight $74.9 \pm 10.36 \mathrm{~kg}$ and height $180.69 \pm 7.14 \mathrm{~cm}$. The subjects performed the test of maximal voluntary contraction (CIVM) of the dominant hand and then, submaximal protocol of ten seconds steps of $20 \%, 40 \%$ and $60 \%$ of CIVM. The proposed test didn't generate a drop on strength performance on the established submaximal steps, as well as the fatigue process establishment of FSD. On the other hand, the FRC showed myoelectric fatigue signs, suggesting stabilizing wrist collapse. These data suggest that myoelectric fatigue of the finger flexors during handgrip is a late process compared to wrist stabilizing fatigue. The protocol in $60 \%$ step seems to trigger the myoelectric fatigue muscle process of the FRC but not the FSD, based on analysis of muscle activation in time and frequency domains.
\end{abstract}

KEY WORDS: Isometry; Dynamometry; Hand; Electromyography.

\title{
Referências
}

1. Esteves AC, Reis DC, Caldeira RM, et al. Força de preensão, lateralidade, sexo e características antropométricas da mão de crianças em idade escolar. Rev Bras Cineantropom Desempenho Hum. 2005;7:69-75.

2. Amaral JF, Mancini M, Novo Júnior JM. Comparação de três dinamômetros de preensão manual relacionados à exatidão e precisão das medidas. Rev Bras Fisiot. 2012;163:216-24.

3. Richards LG. Posture effects on grip strength. Arch Phys Med Rehabil. 1997;78:154-6.

4. Fry AC, Ciroslan D, Fry MD, et al. Anthropometric and performance variables discriminating elite American junior men weightlifters. J Strength Cond Res. 2006;20:861-6.

5. Balogun JA, Akomolafe CT, Amusa LO. Grip strength: effects of testing posture and elbow position. Arch Phys Med Rehabil. 1991;72:280-3.

6. Smith TT, Smith SW, Martin M, et al. Grip strength in relation to overall strength and functional capacity in very old and oldest old females. Phys Occup Ther Geriatr. 2006;24:63-78.

7. Czitrom AA, Lister GD. Measurement of grip strength in the diagnosis of wrist pain. J Hand Surg. 1988;13:16-8.

8. Incel NA, Ceceli E, Durukan PB, et al. Grip strength: effect of hand dominance. Singapore Med J. 2002;43:234-7.

9. Alkurdi ZD, Dweiri YM. A biomechanical assessment of isometric handgrip force and fatigue at different anatomical positions. J Appl Biomech. 2010;26:123-33.

10. Oliveira DG, Aruin AS, Santos MJ. Grip force control in individuals with hand osteoarthritis. J Hand Ther. 2011;24:345-55.

11. Waldo B. Grip strength testing. J Strength Cond Res. 1996;18:32-35.

12. Long C, Conrad PW, Hall EA, Furler SL. Intrinsic-extrinsic muscle control of the hand in power grip and precision handling. J Bone Joint Surg. 1970;52-A:854-67.

13. Ferrer FD. Bases teórico-metodológicas para a preparação física de escaladores desportivos [monografia]. Campinas: Universidade Estadual de Campinas, Curso de Educação Física; 2002.

14. Sahlin K. Metabolic factors in fatigue. Sports Med. 1992;13:99-107.

15. Palmitier RA, An KN, Scott SG, Chao EY. Kinetic chain exercise in knee rehabilitation. Sports Med. 1991;11:402-13.

16. Stuart MJ, Meglan DA, Lutz GE, et al. Comparison of interseg-mental tibiofemoral joint forces and muscle activity during various closed kinetic chain exercises. Am J Sports Med. 1996;24:792-9.

17. Moritani T, Nagata A, Devries HA, Muro M. Critical power as a measure of physical work capacity and anaerobic threshold. Ergonomics. 1981;24:339-50. 
18. Basmajian JV, Deluca CJ. Muscle alive: their function revealed by electromyography. Baltimore: Willians \& Wilkins; 1985. p. 201-22.

19. Dimitrova NA, Dimitrov GV. Amplitude-related characteristics of motor unit and M-wave potentials during fatigue. A simulation study using literature data on intracellular potential changes found in vitro. J Electromyogr Kinesiol. 2002;12:339-49.

20. Dimitrova NA, Dimitrov GV. Interpretation of EMG changes with fatigue: facts, pitfalls, and fallacies. J Electromyogr Kinesiol. 2003;13:13-36.

21. Marson RA. Relationships between surface electromyography and strength during isometric ramp contractions. In: Naik, GR, organizer. Computational intelligence in electromyography analysis: a perspective on current applications and future challenges. Rijeka: Intech; 2012. p. 53-64.

22. Merletti R, Knaflitz M, De Luca CJ. Myoelectric manifestations of fatigue in voluntary and electrically elicited contractions. J Appl Physiol. 1990;69:1810-20.

23. Rondelli RR, Dal Corso S, Simões A, Malaguti C. Métodos de avaliação da fadigabilidade muscular periférica e seus determinantes energético-metabólicos na DPOC. J Bras Pneumol. 2009;35:1125-35.

24. Todor I, Arabadzhiev VG, Dimitrova NA, Dimitrova GV. Interpretation of EMG integral or RMS and estimates of neuromuscular efficiency can be misleading in fatiguing contraction. J Electromyogr Kinesiol. 2010;20:223-32.

25. Jensen B, Laursen B, Sjøgaard G. Aspects of shoulder function in relation to exposure demands and fatigue: a mini review. Clin Biomech. 2000;15:S17-20.

26. Lariviere C, Plamondon A, Lara J, et al. Biomechanical assessment of gloves: study of the sensitivity and reliability of electromyographic parameters used to measure the activation and fatigue of different forearm muscles. Int J Ind Ergonom. 2004;34:101-16.

27. Jackson AS, Pollock ML. Generalized equations for predicting body density of men. Br J Nutr. 1978;40:497-502.

28. Hermens HJ, Freriks B, Merletti R, et al. SENIAM 8: European Recommendations for Surface Electromyography. Roessingh Researchand Development bv, 1999.

29. Field D. Anatomia palpatória. São Paulo: Manole; 2001. p. 46-7.

30. Matsumoto T, Ito K, Moritani T. The relationship between anaerobic threshold and electromyographic fatigue threshold in collegue women. Eur J Appl Physiol. 1991;63:1-5.

31. Mello RGT, Oliveira LF, Nadal J. Digital Butterworth filter for subtracting noise from low magnitude surface electromyogram. Comput Methods Programs Biomed. 2007;87:28-35.

32. Viitasalo JT, Luhtanen P, Rahkila P, Rusko H. Electromyographic activity related to aerobic and anaerobic threshold in ergometer bicycling. Acta Physiol Scand. 1985;124:287-93.

33. Hunter AM, Stclair GA, Lambert M, Noakes TD. Electromyographic (EMG) normalization method for cycle fatigue protocols. Med Sci Sports Exerc. 2002;34:857-61.

34. Hoozemans M, Van Dieën J. Prediction of handgrip forces using surface EMG of forearm muscles. J Electromyogr Kinesiol. 2005;15:358-66.

35. Oliveira LF, Menegaldo LL. Study of muscle torque sharing patterns in isometric plantar flexion by an emg-driven biomechanical model. Annual Meeting of the American Society of Biomechanics; 26-29 aug 2009; Pennsylvania, EUA. Pennsylvania: ASB; 2009. p. 656.

36. Gonzales JU, Scheuermann BW. Gender differences in the fatigability of the inspiratory muscles. Med Sci Sports Exerc. 2006;38:472-9.

\section{Agradecimentos}

Agradecimentos especiais ao Instituto de Pesquisa da Capacitação Física do Exército (IPCFEx) e ao Prof. Runer Augusto Marson pelo apoio na execução desta pesquisa.

Este estudo foi parcialmente financiado pelo CNPq. 
Marco Tulio de Melo Instituto de Pesquisa da Capacitação Física do Exército Fortaleza de São João Av. João Luis Alves, $\mathrm{s} / \mathrm{n}$ 22291-090 - Rio de Janeiro - RJ - BRASIL e-mail: mtulio@peb.ufrj.br; mtulio1993@hotmail.com
Recebido para publicação: 21/01/2013

1a. Revisão: 06/03/2013

2a. Revisão: 01/04/2013

Aceito: 09/04/2013 